\title{
Remarques sur la biologie et l'écologie de Stenasellus virei Dollfus (Crustacea Isopoda Asellota des eaux souterraines)
}

\author{
par
}

\author{
Guy MAGNIEZ*
}

\section{SUMMARY \\ Remarks on the biology and ecology of Stenasellus virei Dollfus (Crustacea Isopoda Asellota of subterranean waters)}

Recent observations indicate that a laying season seems to exist, in karstic as well as in phreatic populations. Nevertheless, a single female cannot lay each year, because the reproductive intermolt averages 15-16 months and is always followed by one (9-10 months) or several non-reproductive intermolts. So, the minimum laying rhythm of $q$ St. virei is biennial.

The cavernicolous population (St. v. virei) of the Padirac swallow-hole is not a relict, but a colony separated from the main settlement of the alluvial waters of the Dordogne river. On the contrary, it is possible to find, close to each other, karstic and phreatic populations which belong to different subspecies (St. v. hussoni and St. v. boui) and live independently.

Les Sténasellides, et plus particulièrement l'espèce polytypique franco-espagnole Stenasellus virei, viennent de faire l'objet d'un travail détaillé, concernant la faunistique, la biologie et l'écologie. Je voudrais revenir sur deux observations intéressantes relatives au problème de la saison et du rythme de ponte d'une part, et au problème des discontinuités faunistiques du domaine aquatique souterrain d'autre part.

1. Saison et rythme de ponte chez St. virei: Il convient de dissocier deux notions qui ne le sont pas toujours:

a) La question de la périodicité de la reproduction à l'échelle des populations: existe-t-il, dans la nature, un maximum saisonnier dans les reproductions, une saison de ponte?

b) La question du rythme des pontes à l'échelle de l'individu: considérant une $q$ qui pond à un moment donné, au bout de combien de temps sera-t-elle capable d'émettre une nouvelle ponte?

* Laboratoire de Biologie Animale et Générale, Université de Dijon, 6, Bd. Gabriel, 21000 Dijon, France. 
a) La réponse à la première question est difficile à généraliser. En effet, bien que plus de 100 stations de l'espèce soient connues, beaucoup sont peu populeuses et n'ont permis que très rarement la capture de $Q$ ovigères. Les seules observations suivies ont été faites à la grotte du Mont-de-Chac en Haute-Garonne où il est possible de recueillir, chaque année, environ une vingtaine de $q$ ovigères (St. v. hussoni). On sait que cette espèce est caractérisée par la durée considérable du développement intramarsupial des embryons, puis des larves (9-10 mois, dans les conditions régnant à la grotte de Moulis). D’une manière générale, lorsque des $q$ ovigères sont capturées dans le gour du Mont-de-Chac à la fin de l'hiver et au printemps (mars-avril), le marsupium contient toujours des oeufs à des stades précoces de segmentation, donc il s'agit de pontes très récentes. Au début de l'été, les $O$ ovigères montrent des embryons à des stades tardifs ou des larves à des stades précoces. A la fin de l'été, période de basses eaux rendant les captures plus faciles, les $O$ ovigères portent très généralement des larves dont le développement est très avancé et dont la naissance est proche.

Ayant placé des $q$ ovigères du. Mont-de-Chac en élevage et noté pour chacune les dates de libération de la portée, il était facile d'en déduire la date approximative de la ponte pour chacune. Il existe nettement une saison de libération des jeunes, qui commence fin octobre et se poursuit en novembre-décembre-janvier, parfois jusqu'à la mi-février. Il lui correspond nécessairement une saison préférentielle de ponte (janvier-avril, dans le cas général), qui paraît centrée sur les mois de février-mars. Des indices donnent à penser que ce phénomène serait très général, aussi bien dans les populations karstiques que phréatiques. Il pourrait être lié au cycle hydrologique du biotope, la période des hautes eaux du début de l'année étant la plus favorable à la mue parturielle des $q$, à l'accouplement et à la ponte.

b) La question du rythme individuel des pontes est très intéressante. Ce rythme est sous la dépendance de celui des mues de la $q$. On sait que les Sténaselles ont une longévité considérable (une quinzaine d'années pour les formes cavernicoles). Leurs intermues durent normalement 9-10 mois et même un an. Les intermues de reproduction des $q$ sont encore plus longues (15-16 mois en moyenne) (fig. 1). De plus, les élevages ont montré que l'intermue de reproduction de la $q$ est toujours suivie d'au moins une intermue de repos sexuel, avec disparition du marsupium.
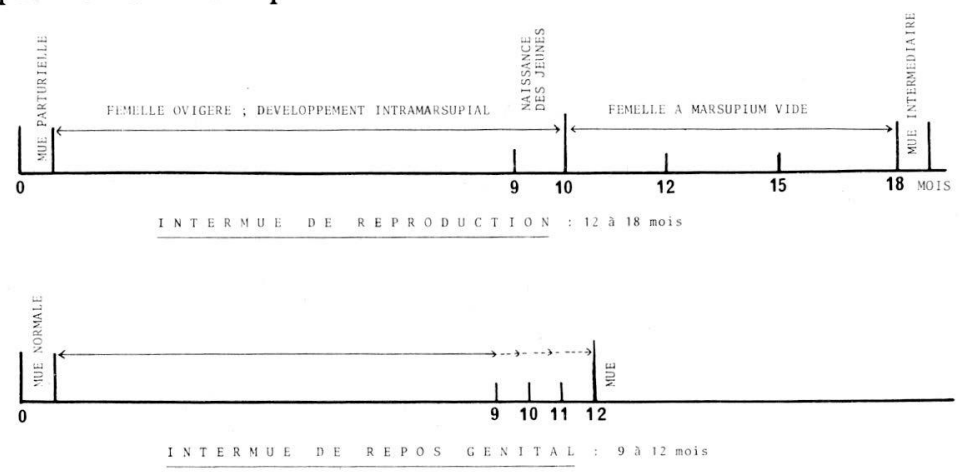

Fig. 1. L'intermue chez la femelle adulte de Stenasellus virei hussoni

Prenons une $q$ donnée, qui pond en janvier-février. Cette $q$ va libérer ses jeunes en octobre-novembre, puis conserver son marsupium vide durant plusieurs mois, avant de subir la mue réductrice des oostégites (en mars-avril de l'année $\mathrm{n}^{0} 2$ ). Elle est donc en intermue de repos génital pour quelques 9-10 
mois. Sa mue suivante, qui pourra être éventuellement une mue reproductrice, ne surviendra pas avant décembre de l'année $n^{0} 2$ ou janvier de l'année $\mathrm{n}^{0}$ 3. Cela signifie qu'une $\circ$ ne peut pas se reproduire tous les ans: le rythme individuel de reproduction est, au minimum, bisannuel (fig. 2). Il semble que

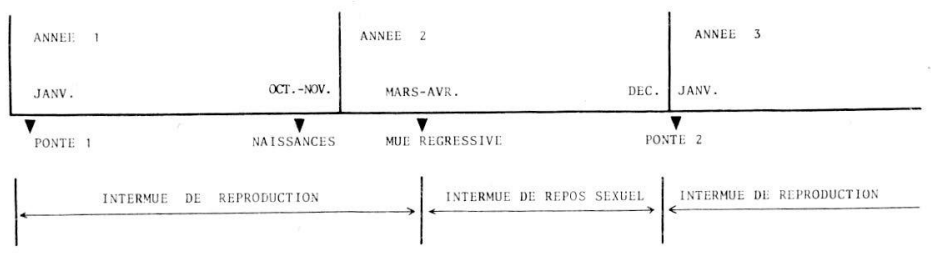

Fig. 2. Périodicité des pontes chez Stenasellus virei hussoni

ce soit le cas général à la grotte du Mont-de-Chac. Dans d'autres stations cavernicoles, caractérisées par des conditions moins favorables (température plus basse, apports nutritifs médiocres), les reproductions seraient plus espacées. Ainsi, on peut estimer qu'à la grotte de l'Estelas, dans l'Ariège, ils s'écoule quelques 5 ans entre deux reproductions successives d'une même $Q$. En d'autres termes, cela signifie que les intermues de reproduction de celle-ci seraient séparées par quelques 4 intermues successives de repos sexuel.

Ainsi, on peut conclure que, chez St. virei, il semble exister une saison de ponte bien marquée, même dans les stations cavernicoles où la température reste très stable tout au long de l'année. Cette synchronisation des reproductions paraît plutôt liée à l'existence d'un cycle hydrologique créant, à la période des hautes eaux, des conditions favorables à la mue, en particulier à la mue parturielle des $q$. Par contre, comme il s'écoule obligatoirement au moins deux ans entre deux pontes successives d'une même $q$, ce ne sont pas les mêmes $q$ qui se reproduisent d'une année à l'autre et il convient de bien séparer, pour cette espèce, les notions de saison de ponte de la population et de rythme individuel de ponte de la $q$.

\section{Continuité et discontinuités du domaine aquatique souterrain habité par}

St. virei: Deux exemples précis sont à citer ici:

a) Sténaselles de Padirac: Le gouffre de Padirac est la station originelle de St. virei où Armand Viré découvrit le Crustacé en 1896. De nombreuses prospections ont eu lieu depuis cette date dans les grottes de cette région des Causses, mais aucune autre station cavernicole n'a été découverte. Ceci avait amené Viré à penser qu'il s'agissait d'une population relique, étroitement confinée dans les eaux de Padirac. Cette population paraîssait largement isolée des peuplements pyrénéens de l'espèce. Or, la forme présente à Padirac est St. v. virei. Cette forme a été, depuis lors, capturée dans les nappes phréatiques et le sous-écoulement du Tarn et de plusieurs autres affluents de la Garonne. Je me suis demandé si cette population cavernicole était réellement isolée ou s'il n'existait pas des populations, géographiquement proches de Padirac, mais installées dans le domaine interstitiel. Or, le gouffre de Padirac est situé à peu de distance de la vallée alluviale de la moyenne Dordogne, qui recoupe les auréoles de calcaires jurassiques du bassin aquitain, avant de pénétrer dans le Massif Central. Des prospections, au moyen de sondages tubés Bou-Rouch, dans les gra- 
viers de la rivière, ont permis la capture de Sténaselles appartenant à la même forme que ceux de Padirac. Depuis les explorations de la rivière souterraine, on sait que les eaux de Padirac parviennent à la Dordogne au niveau d'une grosse résurgence située dans la vallée: la Fontaine de St. Georges à Montvalent (Lot). Nous pouvons donc affirmer que les Sténaselles de Padirac ne constituent pas une population-relique, comme on a pu le penser antérieurement, mais un petit peuplement annexe du peuplement principal colonisant le chenal alluvial de la Dordogne. Il se serait installé dans le milieu karstique grâce à l'existence d'un dispositif hydrogéologique favorable: le fait que l'exutoire du réseau karstique se trouve au niveau même de la nappe fluviale de la Dordogne, permettant un passage aisé de la faune hypogée d'un milieu à l'autre. On sait, par ailleurs, que la rivière souterraine de Padirac est peuplée par l'Aselle oculé Proasellus meridianus. Ce dernier, qui vit normalement à l'extérieur, dans les graviers de la rivière, a pu emprunter le même chemin. Ainsi, dans l'état actuel de nos connaissances, les principaux biotopes de la forme St. v. virei sont constitués par les chenaux d'alluvions récentes du réseau hycrographique de la Garonne, et la population de Padirac ne constitue qu'une curiosité écologique.

b) Sténaselles de la vallée du Lachein: A proximité immédiate du Laboratoire souterrain de Moulis, une petite rivière permanente, affluent du Lez, a encaissé son cours dans un massif karstique crétacé. Ses eaux proviennent pour une part de sources vraies, sur des terrains imperméables et surtout d'une succession de résurgences, étagées le long de la vallée: il s'agit du système karstique du Baget dont l'hydrologie et la faune sont étudiées en détail, respectivement par MM. A. Mangin et R. Rouch, depuis plusieurs années. Les cavités creusées dans la masse calcaire, sur les versants de la vallée (grottes de Ste-Catherine en

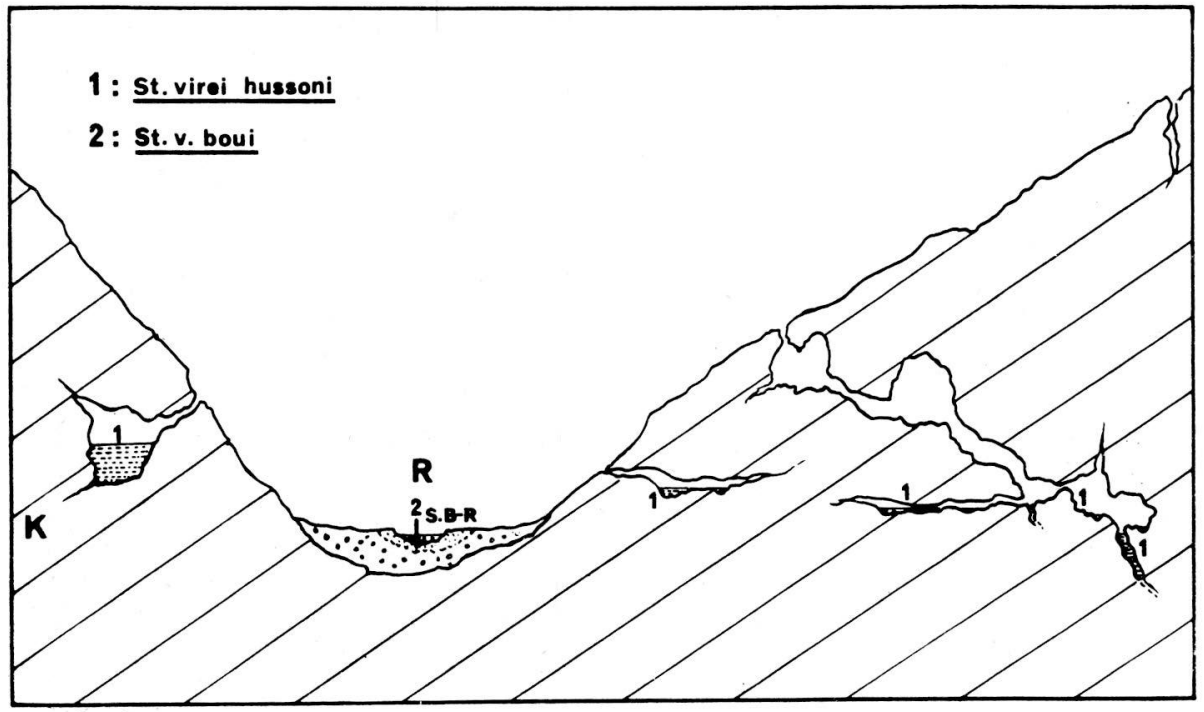

Figure 3: Coupe transversale très schématique dans la vallée du Lachein: $\mathrm{K}=$ massif karstique avec ses cavités et leurs collections d'eau; $\mathrm{R}=$ ruisseau avec sa vallée alluviale; $\mathrm{S}$. $\mathrm{B}-\mathrm{R}=$ sondage tubé BouRouch dans le sous-écoulement (cf. texte). 
particulier), contiennent une faune de St. virei hussoni, forme caractéristique des massifs calcaires nord-pyrénéens situés entre Foix, à l'Est et Bagnères-deBigorre, à l'Ouest. Par ailleurs, les filtrages des différentes résurgences alimentant le ruisseau (Rouch, 1970, 1972) contiennent en général des Sténaselles: il s'agit toujours de cette même forme St. v. hussoni.

Une série de sondages tubés ont été pratiqués, principalement par R. Rouch, dans les alluvions du lit mineur du ruisseau, dans la partie aval de la vallée, là où le cours est pérenne. La plupart ont permis de capturer, parmi une riche faune hyporhéique, quelques Sténaselles: il s'agit toujours de St. virei boui. forme bien distincte de la précédente, et $S t$. v. hussoni paraît totalement absent de ce milieu (fig. 3).

Dans ce second exemple, deux sous-espèces, qui ne dérivent manifestement pas l'une de l'autre, vivent dans des biotopes souterrains très proches, mais de nature différente et il ne semble pas pouvoir s'effectuer d'échanges de faune entre ces deux milieux. On peut alors interpréter le peuplement karstique (St. v. hussoni) comme le plus ancien, alors que le peuplement hyporhéique (St. $v$. boui) paraît résulter d'une colonisation du sous-écoulement du ruisseau à partir de celui du cours d'eau principal, colonisation qui a dû s'opérer à une époque relativement récente. De plus, les conditions hydrogéologiques actuelles semblent telles, qu'elles assurent un certain isolement écologique entre les deux milieux souterrains, contrairement à ce qui existe à l'exutoire de Padirac.

En conclusion, il se pourrait que la distribution locale des Sténaselles dans les eaux souterraines, au sein d'un même réseau hydrographique, soit influencée par la présence ou par l'absence de continuité dans les cheminements souterrains accessibles à ces Crustacés, entre milieux karstiques et milieux phréatiques voisins.

Manuscrit terminé le 9 septembre 1975.

\section{RÉSUMÉ}

Les observations montrent qu'une saison de ponte semble exister aussi bien dans les populations karstiques que phréatiques. Pourtant, une $q$ donnée ne peut pas pondre chaque année, car l'intermue de reproduction dure, en moyenne, 15-16 mois et est toujours suivie d'au moins une (9-10 mois) ou plusieurs intermues de repos génital. Ainsi, le rythme de ponte de St. virei + est, au minimum, bisannuel.

La population cavernicole (St. v. virei) du gouffre de Padirac n'est pas une relicte, mais une colonie adventice, issue du peuplement principal vivant dans la nappe alluviale de la Dordogne. Dans d'autres cas, il est possible de trouver, très près les uns des autres, des peuplements karstiques et phréatiques qui appartiennent à des sous-espèces différentes (St. v. hussoni et St. v. boui) et se maintiennent en toute indépendance.

\section{BIBLIOGRAPHIE}

BOU, Cl., 1974: Les méthodes de récolte dans les eaux souterraines interstitielles. Ann. Spéléol. Fr., 29, 611-619.

LAVAUR, G. de, 1950: Padirac ou l'aventure souterraine. Susse, Paris, 1-112. 
MAGNIEZ, G., 1973: Données récentes sur les Sténaselles (Crustacea Isopoda Asellota) des eaux souterraines continentales. C. R. 96ème Congr. Nat. Soc. Sav. Toulouse, 1971, Sci., 3. 179-191.

- 1974: Observations sur Stenasellus virei dans ses biotopes naturels (Crustacea Isopoda Asellota des eaux souterraines). Int. J. Speleol., 6, 115-171.

1975a: Les stations de Stenasellus virei Dollfus (Crustacé Isopode troglobie), (suite).

Sous le Plancher, Dijon, N.S., 12, 2, 22-26 (1973).
1975b: Observations sur la biologie de Stenasellus virei (Crustacea Isopoda Asellota des eaux souterraines). Int. J. Speleol., 7, 79-228.

VIRÉ, A., 1902: La faune et la flore souterraines du Puits de Padirac (Lot). Bull. Mus. Hist. Nat. Paris, 8, 601-607. 